\title{
Efficiency Benefits Using the Terminal Area Precision Scheduling and Spacing System
}

\author{
Jane Thipphavong ${ }^{1}$ and Harry Swenson ${ }^{2}$ \\ NASA Ames Research Center \\ Moffett Field, California, 94035 \\ Paul $\operatorname{Lin}^{3}$ \\ Optimal Synthesis Inc. \\ Los Altos, California, 94022 \\ Anthony Y. Seo ${ }^{4}$ and Leonard N. Bagasol ${ }^{4}$
University of California, Santa Cruz
NASA Ames Research Center
Moffett Field, California, 94035
}

NASA has developed a capability for terminal area precision scheduling and spacing (TAPSS) to increase the use of fuel-efficient arrival procedures during periods of traffic congestion at a high-density airport. Sustained use of fuel-efficient procedures throughout the entire arrival phase of flight reduces overall fuel burn, greenhouse gas emissions and noise pollution. The TAPSS system is a 4D trajectory-based strategic planning and control tool that computes schedules and sequences for arrivals to facilitate optimal profile descents. This paper focuses on quantifying the efficiency benefits associated with using the TAPSS system, measured by reduction of level segments during aircraft descent and flight distance and time savings. The TAPSS system was tested in a series of human-in-the-loop simulations and compared to current procedures. Compared to the current use of the TMA system, simulation results indicate a reduction of total level segment distance by $50 \%$ and flight distance and time savings by $7 \%$ in the arrival portion of flight $(\sim 200 \mathrm{~nm}$ from the airport $)$. The TAPSS system resulted in aircraft maintaining continuous descent operations longer and with more precision, both achieved under heavy traffic demand levels.

\section{Introduction}

$\mathrm{N}$ EW procedures for the approach and landing phase of flight are being explored to help improve the efficiency of terminal area operations. ${ }^{1}$ This is an integral element of the nation's future air transportation system, known as the Next Generation Air Transportation System (or NextGen), which is envisioned to support an overall increase in air traffic. The Joint Planning and Development Office (JPDO) is responsible for managing the efforts in developing NextGen and its envisioned Concept of Operations (ConOps). ${ }^{2}$ The NextGen goals include the desire to expand airport capacity and protect the environment. One way to achieve these objectives is the shift towards 4-D trajectory-based operations, which allows for more precise trajectory management in timing and position along an aircraft's route. With this capability, more efficient aircraft scheduling and sequencing can be completed, and required spacing between aircraft can be more easily maintained. These, in turn, facilitate Optimal Profile

\footnotetext{
${ }^{1}$ Research Engineer, Aerospace High Density Operations Branch, Mail Stop 210-6, AIAA Member.

${ }^{2}$ Aerospace Engineer, Aerospace High Density Operations Branch, Mail Stop 210-6, AIAA Member.

${ }^{3}$ Research Engineer, 95 First Street, Suite 240.

${ }^{4}$ Senior Software Engineer, Aerospace Simulation Research and Development Branch, Mail Stop 210-6. 
Descents (OPDs) where aircraft descend continuously at near idle thrust from cruise to the airport. OPDs eliminate unnecessary level segments typically used during descent, which reduces fuel consumption, noise and air pollution.

NASA has developed a concept of operations to better manage high-density traffic in the terminal airspace that is directly applicable to NextGen. Core capabilities include extended terminal area routing, precision scheduling along routes, merging and spacing, tactical separation and off-nominal operations recovery. ${ }^{3}$ NASA conducted a series of human-in-the-loop (HITL) simulations to test the feasibility of a mid-term operational concept for these core capabilities, creating the Terminal Area Precision Scheduling and Spacing (TAPSS) system. The TAPSS system is a 4-D trajectory-based strategic and tactical planning and control tool that consists of trajectory prediction, constraint scheduling, runway balancing, controller advisories and flow visualization. ${ }^{4}$ In this concept, arrival aircraft are assigned an Area Navigation (RNAV) Standard Terminal Arrival Route (STAR) prior to top-of-descent (TOD) defined all the way to the runway. The TAPSS system then computes an efficient schedule and arrival sequence for these aircraft to facilitate OPDs. Controllers aim to deliver aircraft to the airport in a smooth, orderly flow using precision metering tools and appropriate speed control to help maintain continuous descents. It is anticipated that doing so reduces complexity of terminal area operations, which in turn helps increase airport throughput without impacting the environment negatively. In fact, human-in-the-loop (HITL) simulation results show that using the TAPSS system over current operations achieves up to a $10 \%$ increase in airport throughput without compromising safety. In addition, TAPSS resulted in better schedule conformance when compared to current operations. ${ }^{4}$

This paper describes results from the same set of simulations, but focuses on quantifying the efficiency benefits of continuous-descent operations using the TAPSS system realized by the reduction of level segments during aircraft descent, along with flight distance and time savings. OPDs have been used operationally at several airports nationwide, but mostly during periods of low to moderate demand (i.e., early morning or late night) due to the procedure possibly interfering with air traffic constraints. ${ }^{5-7}$ The use of the TAPSS system allows for OPDs to be feasible even during high-density traffic demand. There are studies that have documented the benefits of OPDs for various sample sizes, airports and aircraft types. These studies do not take into account explicit arrival scheduling requirements which are especially critical for busy traffic periods. ${ }^{8-15}$ One study has examined the benefits of OPDs under scheduling constraints in high-density terminal airspace. The study compared actual descents with modeled continuous-descent trajectories holding the fixed time-to-fly. ${ }^{16}$ The study, however, does not elaborate on the operational feasibility of conducting OPDs in those conditions. Other NASA research has explored the use of a decision support tool, called Efficient Descent Advisor (EDA), to assist continuous-descent operations from cruise up to the terminal area boundary while taking into account scheduling constraints at the meter fix. ${ }^{17-20}$ EDA has been tested with two aircraft streams under high traffic demand using two consecutive sectors feeding a single meter point situated near the terminal boundary. The TAPSS system, in contrast, provides controller advisories on a larger scale, considering scheduling and sequencing constraints for multiple meter points along aircraft path from cruise to landing to assist arrivals in maintaining continuous descent all the way to touchdown. The major contribution of this study is to investigate energy efficiency benefits achieved when using precision scheduling and spacing from cruise to landing in high-density terminal airspace. The TAPSS system has been tested in HITL simulations that closely model operational use of the concept and moreover, is feasible for the mid-term operational timeframe.

The paper is organized as follows. The next section II further details the TAPSS operational concept and the suite of air traffic management tools employed as part of the TAPSS system. Section III describes the experimental details of the human-in-the-loop simulations conducted to test the concept. Results from the simulations are discussed in section IV, which describe characteristics of the descent profiles, observed flight time/distance savings and a discussion on estimating fuel burn when using the TAPSS system. Lastly, section $\mathrm{V}$ provides a summary of key findings and plans for further research. 


\section{Background}

The TAPSS system is a 4-D trajectory-based strategic and tactical air traffic control (ATC) decision support tool (DST) for managing arrivals in the Terminal Radar Approach Control (TRACON) area. In this concept of operations, arrivals are controlled along a fixed RNAV STAR to a specific runway starting approximately 130-145 $\mathrm{nm}$ from the TRACON boundary. For each aircraft, the TAPSS system uses current state and wind information to determine a 4-D trajectory prediction to the prescribed runway. This is then used as input to compute scheduled times of arrival (STAs) to each meter point: 1) the meter fix located near the TRACON boundary, 2) meter point(s) inside the TRACON and 3) the runway threshold. The arrival times are calculated such that aircraft trajectories are estimated to be conflict-free and satisfy specified separation constraints at the meter points. Demand from other flows at meter points downstream may cause aircraft to be delayed upstream. In this case, the amount of delay is distributed among the arrival route so that controllers can primarily use speed for aircraft control. Controllers are given advisory tools to help meet the STAs to these meter points, while keeping safe separation between aircraft. A general description of the system components is given next for background information. Additional details of the system design and algorithms used can be found in reference 4.

Figure 1 illustrates major functionalities of the TAPSS system and its conceptual relation to the ATC operational systems. The ATC operational systems provide aircraft state information and controller inputs to the TAPSS system as well as display controller advisories received. The main capabilities of the TAPSS system include: flow visualization and control, trajectory prediction, constraint scheduling and runway balancing, and controller advisories. These functionalities are provided by three major component systems: the Traffic Management Advisor (TMA), the Efficient Descent Advisor (EDA) and the controller managed spacing (CMS) tools.

Figure 1. The TAPSS system functional diagram.

TMA is the foundation of the TAPSS system, providing all the main capabilities of the trajectory prediction, constraint scheduling, runway balancing, flow visualization and some of the controller advisories portions. ${ }^{21}$ TMA is currently used operationally at Air Route Traffic Control Centers (or "Centers") nationwide to meter traffic to the TRACON given an airport throughput rate. Meter fixes are typically located close to the TRACON boundary, where an aircraft is issued a de-conflicted STA based on its 4-D trajectory prediction and other scheduling constraints. ${ }^{22}$ TMA also offers Traffic Management Coordinators (TMCs) a variety of flow visualization tools to help determine whether current traffic management initiatives are effective and other actions need to be considered. The original TMA was modified for the TAPSS system by adding more control points along the arrival route that include terminal meter points and the runway threshold. The original TMA scheduler already calculated STAs to the runway threshold, but arrivals were not actively controlled to these arrival times. For the TAPSS system, the TMA scheduler was extended to compute STAs to meter points in the terminal area. Delay needed to meet the STAs at these meter points was distributed across the arrival route segments such that an adequate flow rate to the TRACON was maintained, but did not exceed the limitations of using speed advisories as the primary means for aircraft control. 
EDA provides the Center controller advisories for meeting STAs to the meter fixes. The STAs to the meter fixes are computed by TMA. Controller advisories are designed to be fuel-efficient and conflict-free, thus enabling continuous descents. Figure 2 depicts the EDA advisory panel that is displayed on the Center controller's radar scope for an aircraft, UAL123, heading to the meter fix, SAYGE. Vertical advisories include cruise speed, descent speed and altitude, and horizontal advisories include path stretching. In this case,

UAL123 is advised a cruise mach of 0.76 $(\mathrm{C} / \mathrm{M}$.76) and a descent speed of $250 \mathrm{kts}$

Figure 2. Center Controller Advisory Panel for EDA. (D/250). A path-stretch advisory is also given (@LBF, AMWAY125051, AMWAY), instructing UAL123 to turn at LBF to the place-bearing distance from AMWAY of 125 degrees at 51 miles and then turn directly to AMWAY. In the TAPSS system, this advisory is relayed to pilots via voice command. EDA also provides a meter list and timeline displays for the Center controllers.

The CMS toolset provides the merging and spacing controller support in the TRACON. ${ }^{23-24}$ TMA calculates the STAs to the terminal meter points and uses the CMS algorithms to determine the appropriate speed advisories needed to meet the STAs. Figure 3 shows the different types of controller support tools that can be displayed on the TRACON controller scope. The circular slot markers indicate where an aircraft would be if it were to fly the nominal RNAV arrival route through the forecast wind field, meeting all published restrictions and arriving on time at its STA. Spacing cones also help controllers visually keep aircraft safely separated, with their length varying depending on wake spacing requirements between two aircraft in trail. To follow the slot marker, a speed advisory (e.g. 170 JETSA) is given to the next meter point along the arrival route. As illustrated in Figure 3, ASA278 (on the right side of the figure) is currently flying $210 \mathrm{kts}$ as indicated by the 210 numerical value next to the target symbol ' $\mathrm{C}$ '. The slot marker behind the target symbol indicates that ASA278 should be delayed and is advised to reduce and hold its airspeed at $170 \mathrm{kts}$ until JETSA, a terminal meter point. In the TRACON,

Figure 3. CMS terminal metering, merging and spacing tools. 
STAs generated by TMA are designed to be met using speed advisories alone. In cases where speed advisories would not be sufficient for the aircraft to absorb the delay needed to meet its STA, a late/early indicator (e.g. L 0:30) is displayed. Timelines (not shown) are also available for the controller's use to quickly monitor arrival sequence, current demand loads and delay values.

EDA and CMS were initially developed independently of the TAPSS system and its core functionalities are being continually refined and developed and tested in extensive human-in-the-loop simulations. The enhancements made to TMA (i.e., terminal meter point scheduling) have made it possible to integrate all these DSTs, providing the main capabilities for the TAPSS system. The TAPSS system now offers a fully coordinated ATM solution from the en-route transition to the approach and landing phase of flights, making it easier for controllers to manage continuous-descent operations starting from TOD to the runway threshold.

\section{Experimental Design}

The TAPSS system was tested in a HITL simulation environment using the Multi-Aircraft Control System (MACS) as the simulation platform. ${ }^{25-26}$ MACS provides high-fidelity display emulations for air traffic controllers/managers as well as user interfaces and displays for confederate pilots, experiment managers, analysts, and observers. MACS also has flight deck capabilities that simulate current-day flight technologies that allow controllers to issue ATC clearances. The Aeronautical Datalink and Radar Simulator (ADRS) serves as a communication hub and provides the networking infrastructure that allow numerous MACS operator stations to be connected together. TMA was also connected via the ADRS to provide the necessary information for arrival scheduling, runway assignments, controller advisories and visualization tools.

\section{A. Airspace}

Los Angeles International Airport (LAX) was chosen for the airspace modeling used in the HITL simulations primarily because of its high-traffic density and existing OPD arrival routes. LAX is the sixth busiest airport in the world and a major hub for multiple airlines. ${ }^{27}$ The OPD arrival routes were designed with speed and altitude crossing restrictions that best facilitate OPDs for most aircraft and can easily be loaded into the aircraft's flight management system (FMS). ${ }^{7}$

For the HITL simulations, only arrivals were modeled and operated in the West flow runway configuration with two independently operating runways, 24R and 25L. Figure 4 illustrates the STARs modeled in the simulation for the West flow runway configuration. The current published OPD STARS (i.e., RIIVR and SEAVU) are used by Westbound traffic, accounting for more than $50 \%$ of the arrival traffic. Arrivals on the RIIVR and SEAVU STARs may be assigned to either 24R or 25L as determined by the TMA runway balancing algorithms. The remaining STARS were designed as OPD arrival routes in order to be consistent with the TAPSS operational concept. Approximately a third of the traffic arrives on the SADDE STAR and only uses runway 24R. The rest of the arrivals from the South are always assigned runway $25 \mathrm{~L}$. Arrivals into LAX currently have an aircraft mix of approximately $85 \%$ jets. 
Figure 4. Arrival routes to LAX runway 24R and 25L.

The simulation airspace is segregated into three main areas of control: Los Angeles Center (ZLA), TRACON Feeder and Final. Figure 4 shows the portion of the arrival route each of these areas was responsible for, along with their associated metering point and crossing restrictions. The LA Center sector controllers were responsible for managing the LAX arrivals starting approximately 20 miles before its TOD and ending at its entry into terminal airspace located near the meter fixes, DEANO, PIRUE, GRAMM, KONZL, SHIVE and SXC. For simulation purposes, several of these sectors were combined so that three Center controllers were responsible for the Northwestern (i.e., SADDE), Eastern (i.e., RIIVR, SEAVU) and Southern (i.e., LEENA, SHIVE) STARs. Likewise, three TRACON Feeder controllers handled the next section of the route from the Northwestern (SADDE), Eastern (MINZA, LUVYN) and Southern (MADOW) arrival flows. The last aircraft hand-off is given to one of the two TRACON Final Controllers managing final spacing to LAX runways $24 \mathrm{R}$ and 25L respectively.

\section{B. TMA Scheduler}

As described in the background section, the TMA scheduler outputs STAs at the meter fixes (i.e., DEANO, PIRUE, GRAMM, KONZL, SHIVE, and SXC), terminal meter points (i.e., SADDE, MINZA, LUVYN, MADOW, JETSA and EAGULL) and the runway thresholds (i.e., LAX runways 24R and 25L). The STAs are held fixed or 'frozen' at approximately $135 \mathrm{~nm}$ from LAX, near the Center sector boundary. When applicable, the airport acceptance rate in the scheduler was set to 68 aircraft per hour which is the typical rate used in IFR conditions. When the airport acceptance rate was set, only minimum separation requirements were used as a scheduling constraint. In the case where the airport acceptance rate was set unrestricted, an additional scheduling buffer of $0.4 \mathrm{~nm}$ was added to the required spacing between aircraft. Prior to the HITL simulations, trade studies using fast-time simulations of a generic terminal area arrival scheduler examined the interaction between throughput, delay and controller intervention when varying separation buffers. ${ }^{28}$ Based on these studies and controller feedback, the additional scheduling buffer was chosen such that the number of controller interventions remained at a 
manageable level and meeting the STAs using speed advisories as the primary means of control was achievable in the TRACON airspace.

\section{Scenario}

The simulation scenarios were based on current LAX traffic characteristics with approximately 60 minutes of traffic starting outside the Center boundary. Live LAX traffic data were recorded from March 20-29, 2010 and data from the busiest period was used to determine the aircraft mix, runway demand and STAR usage. Current airport arrival-demand ranged from 55-72 aircraft per hour. According to 2008 Federal Aviation Administration (FAA) Terminal Area Forecast measurements, traffic at LAX is expected to increase $10 \%$ by 2015 and $28 \%$ by $2025 .{ }^{29}$ A scenario was created with current LAX arrivaldemand levels, and three additional scenarios were generated with baseline arrival-demand increasing $5 \%, 10 \%$ and $20 \%$. For each level of demand, three variations of the scenarios were created with different call signs and start times. Thus, a total of 12 scenarios (i.e., 3 scenario variations with 4 demand levels) were created.

\section{Operation Mode}

Two modes of operations were tested in the simulation, referred to as 'TMA' and 'TAPSS.' In the TMA operation mode, used as a baseline for comparison, the original TMA is retained and an airport acceptance rate is specified. Aircraft are metered only to the meter fixes located near the TRACON boundary. Center controllers had the existing TMA meter list and delay advisories, but were not given the explicit EDA advisories (i.e., Figure 2) or timelines. TRACON controllers were not given any of the CMS tools. The TAPSS operation mode, in contrast, included the enhancement made to TMA and did not set an airport acceptance rate. Instead, the separation standards included an additional $0.4 \mathrm{~nm}$ spacing buffer. All the EDA functionalities were available for Center controllers to use. TRACON controllers now controlled to meter points and the runway threshold, with all the CMS tools available for use.

\section{E. Controller and Pilot Tasks}

Eight controllers participated simultaneously to cover all positions. All participants were recently retired (within the previous 2 years) from either SoCal TRACON or Los Angeles Center and had an average of 20 years of ATC experience.

The Center controller responsibilities included assigning the runway and STAR clearance prior to TOD for each aircraft in its sector insuring that the aircraft meet the STA at the meter fix. The runway is chosen such that aircraft delay is minimized. Pseudo pilots entered the STAR into the aircraft FMS display panel along with the appropriate runway. The Center controller then either followed the EDA advisories or used their own technique to control aircraft to meet the meter fix STA. Next, the TRACON Feeder controllers received the aircraft from the Center controller and controlled to the meter points within their sector. Lastly, the Feeder controllers handed off the aircraft to the appropriate TRACON Final controller responsible for proper spacing at the runway. In the TAPSS operation mode, all controllers were advised to use vectoring as a last resort, utilizing speed instructions foremost to manage the arrival traffic.

\section{Results}

The TAPSS system was tested in a series of HITL simulations in the ATC lab at NASA Ames Research Center in the Spring and Fall of 2010. Each series lasted for a period of two weeks where every scenario was run twice. The objectives of the simulation runs in the Spring was to fine tune the parameter settings for the TMA scheduler, validate software processes and provide controller training. Results from the Spring simulation runs can be found in an earlier paper. ${ }^{4}$ The Fall simulation, on the other hand, focused on collecting data to quantify the benefits of using the TAPSS system over the current method of using TMA. These results are presented in this section. 
The energy efficiency benefits were quantified by examining the reduction in level segments during descents and flight path distance and time savings when using the TAPSS system. Minimizing level segments is usually more fuel efficient for aircraft and less controller intervention is needed to issue these advisories. Reducing path distance and time usually implies less fuel burn and delay for the aircraft. For illustration purposes, the scope of this paper shows results for one scenario with its baseline demand level and demand increased 5\% and $10 \%$. These results are representative of the data trends observed in all scenarios used in the simulation.

\section{A. Descent Characteristics}

The descent profiles for the baseline demand increased 5\% for both TMA and TAPSS operations is shown in Figure 5. The vertical profiles for all jet aircraft $(n=70)$ are shown given the along route distance $(\mathrm{nm})$ from the runway threshold. For reference, the approximate location of the final approach fix (FAF), terminal meter point, Center meter fix and freeze horizon relative to the runway threshold are also marked. The aircraft mix in the simulation scenarios includes turboprops, but they are not included in the results since they currently do not conduct OPDs. Comparing Figures 5a and 5b, there are fewer level segments and less variation in the initiation of descent when using the TAPSS system. Most of the level segments occur prior to the meter fix, in Center airspace. Aircraft are also flying higher longer, with TOD points $15 \mathrm{~nm}$ closer to the airport and with half the variance when compared to using only TMA. Later descents may also reduce overall delay since higher speeds are maintained for longer periods of time.

Figure 5. Descent profile for 5\% increase in baseline demand levels for (a) TMA and (b) TAPSS operation modes.

The level segments were identified in the descent profile for each aircraft represented in Figure 5. An algorithm was developed to determine when the level segment occurred along with its length. Details of the algorithm can be found in reference 16. Figure 6 shows for the two operation modes, the total level segment distance summed across all aircraft for the baseline demand and increased levels of 5\% and $10 \%$. The data indicate that the TMA operation mode has more level segments overall. Using TAPSS reduces the total level segment distance $55 \%$ to $85 \%$ than when using TMA alone. Increasing demand levels from the baseline exhibits an increasing number of level-offs during descent. However, when the baseline demand is increased by $10 \%$, the total level segment distance when using TAPSS is still less than using TMA under baseline demand.

The descent profiles were categorized by the meter fix crossing point to determine which routes had the most level segments. Table 1a shows the total level segment distance (nm) for each route via its meter fix when operating in the TAPSS and TMA modes. Data for the baseline demand is shown in the second column, followed by results when increasing the baseline demand by $10 \%$. Table $1 \mathrm{~b}$ lists the average and standard deviation of level segment distance $(\mathrm{nm})$ per aircraft for the increased traffic level of $10 \%$. The percentage change in these tables is calculated by taking the difference between the total level segment 
distances when using the two operation modes, then dividing that value by the total level segment distance when using the TMA operation mode.

Table 1a shows that when using TMA in the baseline demand, longer level segments occur on routes crossing the GRAMM and KONZL (i.e., easternmost) meter fixes which are the RIIVR and SEAVU STARs respectively. Traffic loads on these two STARS are heavy, accounting for $50 \%$ of the LAX demand. When using the TAPSS system, the amount of level segment distance is reduced, by $83 \%-100 \%$ on the RIIVR and

Figure 6. Total aircraft level segment distance $(\mathrm{nm})$ and percentage change when using TAPSS versus TMA under varying demand levels.

SEAVU STARs. On the other hand, two of the routes crossing the PIRUE and SHIVE (i.e., northern and southern) meter fixes show an increase in level segments when using the TAPSS system. This increase in level segment distance (i.e., $+42.36 \mathrm{~nm}$ and $+14.95 \mathrm{~nm}$ ), however, is less than the decrease in level Table 1. (a) Total level segment distance $(\mathrm{nm})$ and percentage change when using TAPSS versus TMA categorized by crossing meter fix. Data is shown for the baseline demand level and increasing traffic levels by $10 \%$. (b) Average and standard deviation of level segment distance $(\mathrm{nm})$ per aircraft when baseline traffic levels increase by $10 \%$.

(a)

\begin{tabular}{|l|c|c|c|c|c|c|c|c|}
\hline Demand Level & \multicolumn{4}{|c|}{ Baseline } & \multicolumn{4}{|c|}{$+10 \%$} \\
\hline Meter Fix & TMA & TAPSS & TAPSS-TMA & $\%$ change & TMA & TAPSS & TAPSS-TMA & $\%$ change \\
\hline \hline DEANO & $0 \mathrm{~nm}$ & $0 \mathrm{~nm}$ & $0 \mathrm{~nm}$ & $0 \%$ & $40.71 \mathrm{~nm}$ & $10.29 \mathrm{~nm}$ & $-30.42 \mathrm{~nm}$ & $-85 \%$ \\
\hline PIRUE & 29.07 & 71.43 & 42.36 & $189 \%$ & 525.78 & 146.43 & -379.35 & $-72 \%$ \\
\hline GRAMM & 342.92 & 56.75 & -286.17 & $-83 \%$ & 239.57 & 311.84 & 72.27 & $30 \%$ \\
\hline KONZL & 206.49 & 0.00 & -206.49 & $-100 \%$ & 267.60 & 11.91 & -255.69 & $-96 \%$ \\
\hline SHIVE & 63.27 & 78.22 & 14.95 & $24 \%$ & 209.52 & 105.83 & -103.69 & $-49 \%$ \\
\hline SXC & 0.00 & 0.00 & 0.00 & $0 \%$ & 4.88 & 0.00 & -4.88 & $-100 \%$ \\
\hline \multicolumn{4}{|c|}{ Average Difference } & $-22 \%$ & \multicolumn{5}{c|}{ Average Difference } & $-43 \%$ \\
\hline
\end{tabular}

(b)

\begin{tabular}{|c|c|c|c|c|c|c|c|c|}
\hline Demand Level & & & & & $+10 \%$ & & & \\
\hline & & & TMA & & & TAPSS & & \\
\hline Meter Fix & $\begin{array}{c}\text { Total } \\
\text { no. ac }\end{array}$ & \begin{tabular}{|c|} 
No. ac \\
w/level \\
segments
\end{tabular} & $\begin{array}{c}\text { Avg level } \\
\text { segment } \\
\text { dist per ac }\end{array}$ & \begin{tabular}{|c} 
St dev of level \\
segment dist \\
per ac
\end{tabular} & $\begin{array}{c}\text { No. ac } \\
\text { w/level } \\
\text { segments }\end{array}$ & \begin{tabular}{|c|} 
Avg level \\
segment \\
dist per ac
\end{tabular} & $\begin{array}{c}\text { St dev of level } \\
\text { segment dist } \\
\text { per ac }\end{array}$ & $\%$ change \\
\hline DEANO & 4 & 1 & $40.71 \mathrm{~nm}$ & $0 \mathrm{~nm}$ & 2 & $5.15 \mathrm{~nm}$ & $1.35 \mathrm{~nm}$ & $-87 \%$ \\
\hline PIRUE & 23 & 15 & 35.05 & 26.00 & 7 & 20.92 & 11.39 & $-40 \%$ \\
\hline GRAMM & 26 & 11 & 21.78 & 28.92 & 7 & 44.55 & 23.55 & $105 \%$ \\
\hline KONZL & 11 & 5 & 53.52 & 35.42 & 2 & 5.96 & 0.78 & $-89 \%$ \\
\hline SHIVE & 10 & 8 & 26.19 & 19.27 & 6 & 17.64 & 18.02 & $-33 \%$ \\
\hline SXC & 1 & 1 & 4.88 & 0.00 & 0 & 0.00 & 0.00 & $-100 \%$ \\
\hline Total & 75 ac & $41 \mathrm{ac}$ & & & $24 \mathrm{ac}$ & Avera & ge Difference & $-13 \%$ \\
\hline
\end{tabular}


segments observed on the routes using the GRAMM and KONZL meter fixes (i.e., -286.17 nm and $206.49 \mathrm{~nm}$ ). These results indicate that some aircraft were advised inefficient maneuvers during their descents; however, there was an overall system benefit.

The routes crossing the PIRUE and SHIVE meter fixes have less traffic, accounting for $20 \%$ of the LAX demand. Since there are different traffic levels for each of the routes, the average percentage difference in level segment distance was weighted by its respective traffic load. Computed this way, the TAPSS system is shown to reduce the amount of level segment distance by $22 \%$ when compared to using TMA solely for the baseline demand level.

When the demand level is increased by $10 \%$, Table 1a shows that using the TAPSS system results in further improvement by reducing the amount of level segments by $43 \%$. Table $1 \mathrm{~b}$ indicates that there are also fewer aircraft performing level-offs during their descents. On a per aircraft basis, there is a large variation in the amount of level segment distance taken. Using the TAPSS system generally reduced the average level segment distance per aircraft, with an overall benefit of $13 \%$.

\section{B. Flight Distance and Time}

The path distance and time for each scenario were also examined from the HITL simulations. The lateral paths of all jets in the scenario with the baseline demand increased by 5\% are shown in Figure 7. Figures $7 \mathrm{a}$ and $7 \mathrm{~b}$ show the results when operating the simulation in TMA and TAPSS modes respectively. The different colors of the lateral paths are for illustrative purposes only. As was also evident from the vertical descent profile plots, most of the vectoring occurs prior to the meter fixes at the Center level. Using TMA alone resulted in more path stretch maneuvers than when using TAPSS. There are a couple of possible explanations for this finding. In the TMA operation mode, the Center controllers made their own judgment on how to best absorb the amount of delay dictated by the scheduler. When using TAPSS, the Center controllers were instead provided with speed and path advisories by the EDA tool. EDA advises speed as the first control method. If speed control is not sufficient to absorb the necessary delay, a path advisory is then given. In the TAPSS operation mode, more speed advisories were used instead of path stretch maneuvers.

Figure 7. Lateral tracks from the HITL simulations for the baseline demand level $+5 \%$ when using (a) TMA and (b) TAPSS. The terminal area is magnified for (c) TMA and (d) TAPSS operation modes respectively. 
Another reason why there are more path stretch maneuvers when using TMA is because the TMA scheduler has a set airport acceptance rate of 68 aircraft per hour, which may restrict the influx of traffic into the terminal area and result in higher delays at the Center level. The higher delays could have resulted in the controllers needing to vector more of the aircraft in order to meet STAs to the meter fix. Using the TAPSS scheduler, the airport acceptance rate is set as unrestricted but the traffic flow is still constrained by the minimum separation standards, additional spacing buffers and scheduling at the runways. These constraints were met with earlier STAs to the meter fix, with less delay to be absorbed in the Center. In this case, Center controllers could mainly use speed advisories to ensure aircraft meet their meter fix STA.

In the terminal area, Figure $7 \mathrm{c}$ highlights the fanning of the paths on the SADDE and SHIVE STARs when using TMA at 5\% demand. Some aircraft are given direct-to-fix instructions in order to fit into the final arrival flow on runway $24 \mathrm{R}$ and $25 \mathrm{~L}$ respectively. This occurs less often when using TAPSS, as seen in Figure 7d. The TAPSS scheduler has computed the schedule in such a way that arrivals remain along their filed lateral path and speed control is used instead to manage traffic. As a result, a more orderly flow is maintained in the terminal area, which facilitates high throughput to the runways.

The distribution of the path distances along each route was further examined. Figure 8 plots the individual path distances $(\mathrm{nm})$ observed for jets on the RIIVR STAR through the GRAMM meter fix, ordered by runway landing time (sec). These are shown for both operation modes, TMA and TAPSS, with the baseline demand level (Figures $8 \mathrm{a}$ and $8 \mathrm{~b}$ ) and when increasing the demand by $10 \%$ (Figures $8 \mathrm{c}$ and d). Under baseline demand levels, using TAPSS does not reduce the path distances. The variation in the path distances is also similar when using either operation mode.

Figure 8. Path distance (nm) by aircraft runway landing time (sec) for the baseline demand and $10 \%$ demand increase for the TMA and TAPSS operating modes. 
As the demand increases, Figures $8 \mathrm{c}$ and $8 \mathrm{~d}$ illustrate longer path distances and larger variation. Using TMA, Figure 8c shows the path distances increasing with later runway landing times. As described earlier, this may be because the arrival rate into the TRACON area was set to 68 aircraft per hour. Since demand to the airport was much higher than this rate, delay accrued at the Center level and affects later aircraft. Moreover, the Center controllers may have utilized more path stretching maneuvers when managing traffic without tools. In contrast, Figure 8d shows that using the TAPSS scheduler results in less path distance variation. Similarly, this was because of the unrestricted rate set in the TAPSS scheduler and more use of speed advisories given by EDA to manage the arrival flow.

Figure 9 shows the average aircraft (a) path distance and (b) time for the RIIVR STAR with increasing demand levels from the baseline to $5 \%$ and $10 \%$. The data show results for the two operation modes, TMA and TAPSS, as well as the "along route" distance and path time for reference. The along route refers to the average path distance and time a jet flies along its filed flight plan without controller intervention. In this case, a jet on the RIIVR STAR flies an average distance $229.6 \mathrm{~nm}$ and has an average time-to-fly of 2233 seconds when flying its nominal route and speed profile. Figure 9a and 9b shows that using TAPSS reduces the average path distance and time compared to using TMA. The benefits of using TAPSS increase as the demand increases.

Figure 9. Path distance (nm) and (b) time (sec) with increasing demand levels for the RIIVR STAR.

The average aircraft path distance and time was computed for the rest of the STARs and is summarized in Table $2 \mathrm{a}$ and $2 \mathrm{~b}$ respectively. The table also presents the along route path distance and time for each of these routes and quantifies the weighted average difference between using TAPSS over TMA. Under baseline traffic levels, the difference between using TAPSS versus TMA is small. There are a few instances where the path distance and time is less than the along route distance and path time, such as those routes going through the DEANO and SHIVE meter fixes. In these cases, the less constrained demand level and larger airspace size of the controlling sector allowed some of the aircraft to shorten their routes using direct-to fix advisories. The weighted average path distance and time difference is 0.24 $\mathrm{nm}$ and 19 seconds respectively. As demand increases, using TAPSS reduces the average path distance and time across most routes with the exception of the LEENA STAR (via SXC). There is less than $1 \%$ of LAX traffic on that route, so the result is from a single jet. Results indicate that when demand levels are increased 10\% from the baseline, TAPSS reduces the average path distance and time difference by 10.52 $\mathrm{nm}$ and 166 seconds respectively. 
Table 2. Average (a) path distance (nm) and (b) time (sec) for each STAR, listed by crossing meter fix, when operating in TMA and TAPSS mode. Results are from using scenarios with baseline demand levels and increasing demand by $10 \%$.

\begin{tabular}{|c|c|c|c|c|c|c|c|c|}
\hline \multirow{2}{*}{$\begin{array}{l}\text { (a) } \\
\text { Meter Fix } \\
\end{array}$} & \multirow{2}{*}{$\begin{array}{c}\text { Demand Level } \\
\text { Along Route } \\
\text { Distance }(\mathrm{nm})\end{array}$} & \multicolumn{3}{|c|}{ Baseline } & \multicolumn{4}{|c|}{$+10 \%$} \\
\hline & & TMA & TAPSS & $\begin{array}{c}\text { Difference } \\
\text { (TAPSS-TMA) }\end{array}$ & $n$ & TMA & TAPSS & $\begin{array}{c}\text { Difference } \\
\text { (TAPSS-TMA) }\end{array}$ \\
\hline DEANO & 250.74 & 244.47 & 243.78 & -0.69 & 4 & 258.32 & 251.36 & -6.96 \\
\hline PIRUE & 224.89 & 225.94 & 225.62 & -0.32 & 23 & 236.37 & 227.41 & -8.96 \\
\hline GRAMM & 229.60 & 229.51 & 229.23 & -0.27 & 26 & 249.41 & 232.28 & -17.13 \\
\hline KONZL & 241.61 & 241.67 & 241.97 & 0.30 & 11 & 254.53 & 243.06 & -11.47 \\
\hline SHIVE & 196.49 & 190.45 & 194.39 & 3.94 & 10 & 197.93 & 196.26 & -1.67 \\
\hline SXC & 250.65 & 250.70 & 250.76 & 0.06 & 1 & 248.39 & 250.67 & 2.28 \\
\hline & & \multicolumn{2}{|c|}{ Average Difference } & $0.24 \mathrm{~nm}$ & \multicolumn{3}{|c|}{ Average Difference } & $-10.52 \mathrm{~nm}$ \\
\hline \multirow{2}{*}{\begin{tabular}{|l|} 
(b) \\
Meter Fix \\
\end{tabular}} & Demand Level & \multicolumn{3}{|c|}{ Baseline } & \multicolumn{4}{|c|}{$+10 \%$} \\
\hline & $\begin{array}{c}\text { Along Route } \\
\text { Path Time (sec) }\end{array}$ & TMA & TAPSS & $\begin{array}{c}\text { Difference } \\
\text { (TAPSS-TMA) } \\
\end{array}$ & \multicolumn{2}{|r|}{ TMA } & TAPSS & $\begin{array}{c}\text { Difference } \\
\text { (TAPSS-TMA) }\end{array}$ \\
\hline DEANO & 2388 & 2309 & 2343 & 34 & \multicolumn{2}{|r|}{2693} & 2460 & -233 \\
\hline PIRUE & 2227 & 2221 & 2223 & 2 & \multicolumn{2}{|r|}{2542} & 2279 & -263 \\
\hline GRAMM & 2233 & 2272 & 2270 & -2 & \multicolumn{2}{|r|}{2510} & 2337 & -173 \\
\hline KONZL & 2373 & 2366 & 2388 & 22 & \multicolumn{2}{|r|}{2532} & 2423 & -109 \\
\hline SHIVE & 1996 & 1872 & 1918 & 46 & \multicolumn{2}{|r|}{2008} & 1984 & -24 \\
\hline SXC & 2360 & 2252 & 2319 & 67 & \multicolumn{2}{|c|}{\begin{tabular}{l|l}
2305 & \\
\end{tabular}} & 2327 & 22 \\
\hline & & Average [ & ifference & $19 \mathrm{sec}$ & & verage $\mathrm{Di}$ & ifference & $-166 \mathrm{sec}$ \\
\hline
\end{tabular}

\section{Fuel Benefits}

Previous sections examined the number of level segments used during descent and the path distance and time for each of the routes. These metrics were evaluated for the two operation modes, TMA and TAPSS, and for increasing demand levels. Using TAPSS generally reduced the number of level segments and path distance and time overall. Intuitively, this should translate to less fuel consumption. Quantifying the amount of fuel consumption, however, is not a straightforward process. A complete fuel consumption analysis of this simulation data should ideally include both a description of fuel burn during level segments as well as the descent portion of the flight. Such an analysis is best achieved when the simulation platform contains an internal fuel burn model. The simulation platform utilized in this study (MACS) does not have its own internal fuel burn model, though uses thrust and drag models for each of the aircraft simulated. These models have some variation from other simulation tools such as the Boeing Flight Operations Engineering inflight performance data (INFLT) ${ }^{30}$ and the Eurocontrol's Base of Aircraft Data (BADA). ${ }^{31}$ Two problems exist when trying to use these other fuel burn models with the MACS simulation results. First, to infer the fuel burn from MACS data when an aircraft is cleared for descent, the MACS FMS model calculates the fuel-efficient descent speed depending on the aircraft type. These computed speeds may not be considered fuel-efficient according to other models, instead resembling powered flight even when the trajectory is flight-idle. Secondly, the fuel burn saving is a small value in the first place. Attempting to use the MACS simulation data with these other simulation systems resulted in inconclusive results. Due to the absence of a complete fuel burn model in the simulation software, a partial analysis of the fuel consumption benefits was performed on the simulation data, examining the level segments only. 
This fuel consumption calculation was based on the assumption that the speed of the level segments is issued by the controller and not determined by the MACS FMS. Prior research has reconciled the differences between various fuel burn models and compared the BADA fuel flow predictions and published values from the aircraft operating manuals of several Boeing and Airbus aircraft; the study found that BADA tends to under-predict fuel flow by 15$25 \% .{ }^{11}$ The BADA fuel flow model was modified to correct these errors and was then used to

Figure 10. Fuel burn (kg) for total level segment distance when using TMA and TAPSS under varying demand levels. calculate the fuel burn for the total level segment distances depicted in Figure 6, and the results are plotted in Figure 10. The plot exhibits a similar trend as seen in Figure 6, where using TMA results in greater fuel consumption since there are more level segments in the aircraft descents. However, note that in Figure 10, the percentage difference between using TAPSS versus TMA is not the same as Figure 6. In the cases of baseline traffic demand levels and increasing it to $5 \%$, the percentage difference is less than shown in Figure 6. This is due to the fuel flow rate not having a linear relationship with altitude and speed.

\section{Conclusion and Next Steps}

NASA has developed a capability for terminal area precision scheduling and spacing (TAPSS) to increase the use of fuel-efficient arrival procedures during periods of traffic congestion at a high-density airport. The TAPSS system is a 4D trajectory-based strategic and tactical planning and control tool that computes scheduling and sequencing for arrivals to facilitate continuous-descent approaches during high traffic demand periods. The TAPSS system was tested in a series of HITL simulations that closely model operational use of the concept for the mid-term NextGen timeframe. The energy efficiency benefits when using the TAPSS system over the current use of the TMA system was examined in one simulation. Actual fuel burn was not quantified due to the inconsistency among different fuel flow models. Efficiency benefits were instead measured by the reduction of level segments during aircraft descent and flight distance and time savings. Compared to the current use of the TMA system, simulation results indicate a reduction of total level segment distance by $50 \%$ and flight distance and time savings by $7 \%$ in the arrival portion of flight ( $200 \mathrm{~nm}$ from the airport). The TAPSS system resulted in aircraft maintaining continuous descent operations longer and with more precision; both these objectives were determined to be achievable under heavy traffic demand levels.

Future research will exercise the robustness of the TAPSS system. Developing procedures to deal with off-nominal situations such as missed approaches will be studied. Experiments will also examine the integration of flight deck precision spacing capabilities. These efforts will begin to establish the concept of operations and procedures necessary to handle the controller and pilot interactions in a mixed equipage environment. Results from these HITL simulations eventually supports a field demonstration of the integration of advanced time-based scheduling with controller- and flight deck-based precision spacing capabilities. 


\section{References}

1 Federal Aviation Administration. (2010, May 3). Descent, Approach and Landing Benefits. Retrieved December 07, 2010,

from http://www.faa.gov/nextgen/benefits/descent/

2 Joint Planning and Development Office. (2010). Concept of Operations for the Next Generation Air Transportation System (Version 3.2 ed.). Washington, DC.

3 Isaacson, D. R., Swenson, H. N., \& Robinson III, J. E. (2010). A Concept for Robust, High Density Terminal Air Traffic Operations. 10th AIAA Aviation Technology, Integration, and Operations Conference (ATIO). Fort Worth, TX.

4 Swenson, H. N., Thipphavong, J., Sadovsky, A., Chen, L., Sullivan, C., \& Martin, L. (2011). Design and Evaluation of the Terminal Area Precision Scheduling and Spacing System. Ninth USA/Europe Air Traffic Management Research and Development Seminar. Berlin, Germany.

5 Clarke, J. P., Bennett, D., Elmer, K., Firth, J., \& Hilb, R. (2006). Development, design, and flight test evaluation of a continuous descent approach procedure for nighttime operation at Louisville International Airport. Partnership for Air Transportation Noise and Emissions Reduction An FAA/NASA/Transport Canada-Sponsored Center of Excellence.

6 Staigle, T., \& Nagle, G. (September 6-7, 2006). Details and Status of CDA Procedures for Early Morning Arrivals at Hartsfield-Jackson Atlanta International Airport (ATL). CDA Workshop No. 3. Atlanta, GA: Georgia Institute of Technology.

7 White, W. (February 23, 2009). SoCal Optimized Profile Descent Development. Presented to NASA Ames Research Center. Airspace and Procedures, Southern California TRACON.

8 Alcabin, M., Schwab, R. W., Soncrant, C., Tong, K. O., \& Cheng, S. S. (Sept. 21-23, 2009). Measuring Vertical Flight Path Efficiency in the National Airspace System. 9th AIAA Aviation Technology, Integration, and Operations Conference (ATIO). Hilton Head, SC.

9 Dinges, E. (July 2-5, 2007). Determining the Environmental Benefits of Implementing Continuous Descent Approach Procedures. 7th USA/Europe Seminar on Air Traffic Management Research and Development (ATM 2009). Barcelona, Spain.

${ }^{10}$ Melby, P., \& Mayer, R. H. (Sept. 14-19, 2008). Benefit Potential of Continuous Climb and Descent Operations. The 26th Congress of International Council of Aeronautical Sciences (ICAS). Anchorage, AK.

${ }^{11}$ Reynolds, T. G. (Jun.29 - Jul. 2, 2009). Development of Flight Inefficiency Metrics for Environmental Performance Assessment of ATM. 8th USA/Europe Seminar on Air Traffic Management Research and Dvelopment (ATM2009). Napa, CA.

${ }^{12}$ Shresta, S., Neskovic, D., \& Williams, S. S. (Jun. 29 - Jul. 2, 2009). Analysis of Continuous Descent Benefits and Impacts During Daytime Operations. 8th USA/Europe Seminar on Air Traffic Management Research and Development (ATM2009). Napa, CA.

${ }^{13}$ Sprong, K. R., Klein, K. A., \& Shiotsuki, C. (Oct. 26-30, 2008). Analysis of AIRE Continuous Descent Operations at Atlanta and Miami. 27th Digital Avionics Systems Conference (DASC), (pp. 3.A.5-1-3.A.5-13). St. Paul, MN.

${ }^{14}$ Tong, K. O., Boyle, D. A., \& Warren, A. W. (Sept. 25-27, 2006). Development of Continuous Descent Arrival (CDA) Procedures for Dual-Runway Operations at Houston Intercontinental. 6th AIAA Aviation Technology, Integration, and Operations Conference (ATIO). Wichita, KS.

${ }^{15}$ Wat, J., Follet, J., Mead, R., \& Brown, J. (Sept. 25-27, 2006). In Service Demonstration of Advanced Arrival Techniques at Schipol Airport. 6th AIAA Aviation Technology, Integration, and Operations Conference (ATIO). Wichita, KS.

${ }^{16}$ Robinson, J. E., \& Kamgarpour, M. (Sept. 13-15, 2010). Benefits of Continuous Descent Operations in High-Density Terminal Airspace Under Scheduling Constraints. 10th AIAA Aviation Technology, Inegration, and Operations (ATIO) Conference. Fort Worth, TX.

${ }^{17}$ Coppenbarger, R., Dyer, G., Hayashi, M., Lanier, R., Stell, L., \& Sweet, D. (2010). Development and Testing of Automation for Efficient Arrivals in Constrained Airspace. 27th International Congress of the Aeronautical Sciences. Nice, France.

${ }^{18}$ Coppenbarger, R., Lanier, R., Sweet, D., \& Dorsky, S. (2004). Design and Development of the En-Route Descent Advisor (EDA) for Conflict-Free Arrival Metering. AIAA Guidance, Navigation and Control (GNC) Conference. Providence, RI.

${ }^{19}$ Coppenbarger, R., Mead, R., \& Sweet, D. (2007). Field Evaluation of the Tailored Arrivals Concept for Datalink-Enabled Continuous Descent Approach. 7th AIAA Aviation Technology, Integration and Operations (ATIO) Conference. Belfast, Northern Ireland.

${ }^{20}$ Sweet, D., Dorsky, S., \& Mueller, T. (Oct. 2004). Data Analysis Report for the July, 2004 En Route Descent Advisor Simulation. Sunnyvale, CA: Seagull Technology, Inc.

${ }^{21}$ Swenson, H. N., Hoang, T., Engelland, S., Vincent, D., Sanders, T., Sanford, B., et al. (1997). Design and Operational Evaluation of the Traffic Management Advisor at the Fort Worth Air Route Traffic Control Center. 1st USA/Europe Air Traffic Management Research and Development Seminar. Saclay, France.

${ }^{22}$ Wong, G. (2000). The Dynamic Planner: The Sequencer, Scheduler, and Runway Allocator for Air Traffic Control Automation. Moffet Field: NASA.

${ }^{23}$ Callantine, T. J., \& Palmer, E. A. (Sept. 21-23, 2009). Controller Advisory Tools for Efficient Arrivals in Dense Traffic Environments. 9th AIAA Aviation Technology, Integration, and Operations Conference (ATIO). Hilton Head, South Carolina.

${ }^{24}$ Kupfer, M., Callantine, T. J., Mercer, J., Martin, L., \& Palmer, E. (Aug. 2-5, 2010). Controller-Managed Spacing--A Human-In-The-Loop Simulation of Terminal-Area Operations. AIAA Guidance, Navigation, and Control Conference. Toronto, Ontario.

${ }^{25}$ Prevot, T., Callantine, T., Lee, P., Mercer, J., Palmer, E., \& Smith, N. (July 2004). Rapid Prototyping and Exploration of Advanced Air Traffic Concepts. International Conference on Computational and Engineering Science. Madeira, Portugal. 
${ }^{26}$ Prevot, T. M. (August 2007). MACS: A Simulation Platform for Today's and Tomorrow's Air Traffic Operations. AIAA Modeling and Simulation Technologies Conference and Exhibit. Hilton Head, SC.

${ }^{27}$ Anon. (n.d.). Los Angeles International Airport. Retrieved June 20, 2011, from Wikipedia: http://en.wikipedia.org/wiki/Los_Angeles_International_Airport

${ }^{28}$ Thipphavong, J., \& Mulfinger, D. (Sept. 13-15, 2010). Design Considerations for a New Terminal Area Arrival Scheduler. AIAA 10th Aviation Technology, Integration, and Operations (ATIO) Conference. Fort Worth, TX.

${ }^{29}$ Gawdiak, Y. (2008). JPDO Systems Modeling and Analysis Division (SMAD): NextGen Airport Capacity Benefits Analysis.

${ }^{30}$ The Boeing Company Flight Operations Engineering. (1999). INFLT and REPORT Software. Seattle, WA.

${ }^{31}$ EUROCONTROL Validation Infrastructure Centre of Expertise. (March 2009). User manual for the base of aircraft data (BADA). France. 